\title{
Caractérisation Physico-Chimique Et Étude De La Toxicité De La Lagune Côtière, De Togbin À Grand- Popo (Sud-Ouest Bénin)
}

\author{
Waris Kéwouyèmi Chouti \\ Laboratoire d'Hydrologie Appliquée, Institut National de l'Eau (INE), \\ Université d'Abomey-Calavi, Cotonou, Bénin \\ Laboratoire de Chimie Inorganique et de l'Environnement, Faculté des \\ Sciences et Techniques (FAST), Université d'Abomey-Calavi, Cotonou, \\ Bénin \\ Nafiou Egbéola Chitou \\ Laboratoire de Chimie Inorganique et de l'Environnement, Faculté des \\ Sciences et Techniques (FAST), Université d'Abomey-Calavi, Cotonou, \\ Bénin \\ Nelly Kelome \\ Boris Boniface H. Kpako \\ Dorcas Honvou Vlavonou \\ Michael Tossou \\ Laboratoire d'Hydrologie Appliquée, Institut National de l'Eau (INE), \\ Université d'Abomey-Calavi, Cotonou, Bénin
}

doi: 10.19044/esj.2017.v13n27p131 URL:http://dx.doi.org/10.19044/esj.2017.v13n27p131

\begin{abstract}
This work deals with the physicochemical characterization and the toxicity study of the coastal lagoon of Benin (from Togbin to Grand-Popo). In particular, it has to do with the study of copper and zinc. The sampling campaign was carried out by motorized boat on the $20^{\text {th }}$ of December 2016. Samples were taken a few centimeters below the surface of the water. The analysis of the metals was carried out with a HACH LANGE DR2800 spectrophotometer. Copper is determined by the method of Bicinchroninate, while zinc is determined by the method of Zincover. The method used for the toxicity study is natural and is based on the inoculation of onions in the waters sampled at various proportions. The results revealed that physically and chemically, the lagoon is highly mineralized. This mineralization is due not only to the contribution of the sea to high tide, but also to salt production in Djègbadji. Concerning the concentrations of trace metallic elements, copper and zinc do not pose a risk to humans, but pose a high risk to the
\end{abstract}


aquatic organisms present in the lagoon. Potentially suspected sources of copper and zinc are Ahémé Lake and the Mono River. As for the ecotoxicological study with the Allium cepa test, this test is not only influenced by metals, but also by the salinity of the water. The test revealed that the waters of the lagoon are acutely toxic, particularly at Djondji. Strategies for the protection and rehabilitation of the coastal lagoon must be undertaken by decision-makers at all levels. This, therefore, will help to prevent not only the different populations from the risks of diseases, but also increase the economy of the country through marketing and the edibility of the fish and even offer a framework of tourist scale.

Keywords: Toxicity, metals, copper, zinc, écotoxicological, Allium cepa

\section{Resume}

Ce travail porte sur la caractérisation physico-chimique et l'étude de toxicité de la lagune côtière du Bénin (de Togbin à Grand-Popo), notamment l'étude du cuivre et du zinc. La campagne d'échantillonnage a été effectuée en barque motorisée le 20 décembre 2016. Les échantillons ont été prélevés à quelques centimètres sous la surface de l'eau. L'analyse des métaux a été effectuée avec un spectrophotomètre HACH LANGE DR2800. Le cuivre est déterminé par la méthode de Bicinchroninate et le zinc par la méthode de Zincover. La méthode utilisée pour l'étude de toxicité est naturelle et se base sur l'ensemencement d'oignons dans les eaux échantillonnées à diverses proportions données. Les résultats ont révélé que, sur le plan physicochimique, la lagune est fortement minéralisée et cette minéralisation est due non seulement à l'apport de la mer en haute marrée mais aussi à la production de sel à Djègbadji. Concernant les concentrations des éléments traces métalliques, le cuivre et le zinc, ne constituent pas un risque pour l'Homme mais représentent un risque élevé pour les organismes aquatiques présents dans la lagune. Les sources de cuivre et de zinc potentiellement soupçonnées sont le lac Ahémé et le fleuve Mono. Quant à l'étude écotoxicologique avec le test sur l'Allium cepa, ce test est non seulement influencé par les métaux mais aussi par la salinité de l'eau. Le test a révélé que les eaux de la lagune présentent une toxicité aiguë et particulièrement à Djondji. Des stratégies de protection et d'assainissement de la lagune côtière doivent être entreprises par les décideurs à tous les niveaux, ce qui permettra d'éviter non seulement aux différentes populations des risques de maladies mais aussi d'augmenter l'économie du pays par la commercialisation et la comestibilité des poissons et même d'offrir un cadre d'envergure touristique.

Mots-clés : Toxicité, métaux, cuivre, zinc, écotoxicologique, Allium cepa 


\section{Introduction}

La compréhension, l'exploitation raisonnée et la conservation des écosystèmes aquatiques sont devenues des enjeux écologique et économique considérables et sont aujourd'hui au centre des politiques de développement durable, tant nationales qu'internationales. Ainsi, au titre du rapport 2015 des Objectifs du Millénaire pour le Développement (OMD), il a été souligné la nécessité de s'assurer que le programme de développement pour le futur reflète les liens entre viabilité socioéconomique et environnementale et qu'il protège et renforce l'environnement.

Le Bénin, à l'instar des Pays en Développement est touché par une explosion démographique entraînant une occupation anarchique des sols (Alassane, 2004) suivie de multiples activités anthropiques qui drainent constamment et fréquemment des flux de production et de consommation, qui ont pour corollaire des rejets de substances polluantes dans l'environnement et notamment dans les eaux superficielles. Compte tenu de son statut de réceptacle final, le milieu aquatique regroupe les écosystèmes les plus touchés, directement ou indirectement, par les effets délétères des polluants (Manar, 2008). De plus, plusieurs de ces contaminants sont persistants et bioaccumulables, ce qui signifie qu'ils peuvent entraîner des effets nuisibles au niveau de tous les maillons de la chaîne alimentaire, incluant la faune terrestre et aviaire ainsi que l'humain, du fait que les contaminants sont transmis d'un maillon à l'autre par la consommation d'organismes aquatiques contaminés (MDEP, 2009). Pour l'être humain, la pollution bactérienne est la plus dangereuse ; ensuite vient la pollution chimique toxique, qui, aujourd'hui constitue aussi la plus grande menace à la comestibilité des poissons et à la qualité des eaux potables (Adrian et al., 2006). Parmi les contaminants majeurs de l'environnement, les métaux posent de sérieux problèmes écologiques, tant par le caractère ubiquiste de leur présence au sein de la biosphère que par leur forte rémanence et leur toxicité élevée (Barillet, 2007). Ainsi, les eaux de surface sont exposées aux effets toxiques des polluants modifiant la qualité physico-chimique de ces eaux.

Contrairement aux analyses chimiques qui donnent les concentrations de différents polluants dans un échantillon d'eau, les tests d'écotoxicité permettent d'évaluer l'effet, peu importe le polluant, en mettant en contact l'échantillon avec des organismes. Ces tests renseignent sur la biodisponibilité des polluants, c'est-à-dire s'ils sont assimilables par les organismes vivants, et si ces polluants peuvent les affecter. Il est à noter que les analyses chimiques et les tests d'écotoxicité se complètent. Les plantes constituent un élément essentiel des chaînes trophiques. En outre, elles représentent également un outil formidable pour une meilleure compréhension des risques environnementaux auxquels la population 
humaine est exposée (Ma et al., 1995) et présentent une structure chromosomique identique à celle des mammifères (Cotelle, 1999). Les bioessais couramment utilisés en écotoxicologie et normalisés au niveau international, tels que le test plante, se sont avérés efficaces pour révéler la toxicité des eaux. Le réseau racinaire des plantes possède une surface d'échange phénoménale, ce qui les rend particulièrement vulnérables aux polluants (Foltete, 2010). L'utilisation des plantes comme modèle expérimental pour évaluer la toxicité est relativement attrayante puisque ces tests sont relativement simples à réaliser, peu coûteux, sensibles, et rapides (Goujon, 2015). Les modèles végétaux utilisés dans les tests de génotoxicité sont le plus souvent : Allium cepa, Tradescantia et Vicia faba. Le test d'Allium cepa a été utilisé par nombreux auteurs (Riffat \& Ahmad, 2006; Mittergger et al., 2007; Feretti et al., 2007; Leme \& Marin-Morales, 2008; Mustafa \& Arikan, 2008; Fasla, 2009; Ashraf \& Husain, 2010; Halmi, 2015). Ces auteurs ont montré que l'Allium cepa est très sensible pour l'évaluation de la biodisponibilité des contaminants. Les bulbes à tunique concentrique des divers Allium cepa sont plus ou moins riches en eau car sa teneur en matière sèche varie de 7 à 15\% (Bedouh, 2014). Les critères utilisés dans ces tests sont non seulement des critères généraux (germination, croissance) mais aussi génotoxiques (toxicité des contaminants environnementaux vis-à-vis du matériel génétique des êtres vivants). Ces critères permettent d'évaluer les effets des micropolluants sur les êtres vivants.

Les lagunes côtières sont connues comme des nurseries et des milieux très productifs en raison de facteurs physiques très variables, de l'hétérogénéité spatiale des structures des peuplements fortement liée à la disponibilité des ressources alimentaires (Villanueva, 2004). Malheureusement, la lagune côtière du Bénin est sujette à nombreux cas de pollution anthropique. Il s'agit notamment des dépotoirs sauvages d'ordure au niveau des rives de la lagune, le ruissellement des eaux usées dans la lagune ainsi que le rejet direct des fèces dans la lagune ou par l'intermédiaire des latrines non étanche. La lagune côtière est également soumise à une dynamique hydrologique caractérisée par des apports en eau salée de l'océan Atlantique. En effet, soucieux de compléter les informations sur la qualité des eaux de la lagune côtière du Bénin, en dépit des différentes études déjà menées sur ce milieu qui, néanmoins, n'ont pas abordé le caractère écotoxique basé sur l'Allium cepa, notre travail s'inscrit dans cette thématique globale et concerne en particulier l'étude physico-chimique des eaux de la lagune, et parallèlement, une approche de mesure de la toxicité des eaux de la lagune pour les organismes aquatiques sera évaluée par le test des oignons. 


\section{Materiel et methodes}

\section{Présentation du milieu d'étude}

La lagune côtière, longue d'environ $65 \mathrm{~km}$, d'une superficie de 55 $\mathrm{km}^{2}$ est la plus grande lagune du Bénin. Encore appelée le Djessin, il s'étend de la partie occidentale de Cotonou jusqu'à Hlihouè sur le littoral du pays. Ce plan d'eau pérenne comprend deux sections qui sont : la section orientale, ou lagune de Ouidah, qui communique avec l'embouchure du fleuve Mono, et la section ouest où on retrouve la lagune de Grand-Popo. La lagune Djessin collecte les eaux des rivières côtières, du fleuve Mono, du défluent Sazué, du Couffo, et assure leur transport dans l'océan Atlantique à Vodounonkodji (Fiogbé et al., 2007). Les profondeurs dans la lagune côtière sont relativement faibles. En basses eaux, elles varient entre $0,4 \mathrm{~m}$ et $1,70 \mathrm{~m}$ et en hautes eaux entre $1,10 \mathrm{~m}$ et $3,40 \mathrm{~m}$. Néanmoins, la profondeur de la lagune côtière est relativement importante à l'Ouest de son embouchure dans la mer, de 4 à $5 \mathrm{~m}$ en saison de pluies (PGPE, 2008). Elle jouit d'un climat soudanoguinéen, caractérisé par deux saisons de pluie alternée de deux saisons sèches à durées inégales. La lagune côtière entre Grand-Popo et Togbin est alimentée par trois fleuves venant du Nord : le Mono et son affluent la Sazué, ainsi que le Couffo qui se jette dans le lac Ahémé, celui-ci communiquant avec la lagune par l'intermédiaire de l'Aho.

\section{Prélèvement des échantillons}

La sélection des sites d'échantillonnage est faite selon un choix raisonné en considérant à la fois la densité de la population riveraine, les activités anthropiques aux environs et dans la lagune, les limites des villages et la portée de chaque site de prélèvement. La campagne d'échantillonnage a été effectuée en barque motorisée. Les échantillons ont été prélevés à quelques centimètres sous la surface de l'eau. Des bouteilles en plastique de 1,5 litre et des bidons de 20 litres ont été remplies après 3 rinçages avec l'eau à échantillonner. Au total, 06 sites ont été retenus et présentés sur la Figure 1.

\section{Procédure d'analyse physico-chimique}

Le potentiel d'hydrogène $(\mathrm{pH})$ et le potentiel redox $\left(\mathrm{E}^{\circ}\right)$ sont mesurés par un pH-mètre de marque WTW 3110. La température, la conductivité, les solides totaux dissous (TDS) et la salinité sont mesurés par un conductimètre de marque WTW 3210. Pour chaque mesure, la sonde est d'abord rincée avec l'eau distillée et ensuite avec l'échantillon. La sonde a été ensuite plongée dans l'échantillon d'eau prélevé et les différents paramètres sont lus et consignés in-situ. 


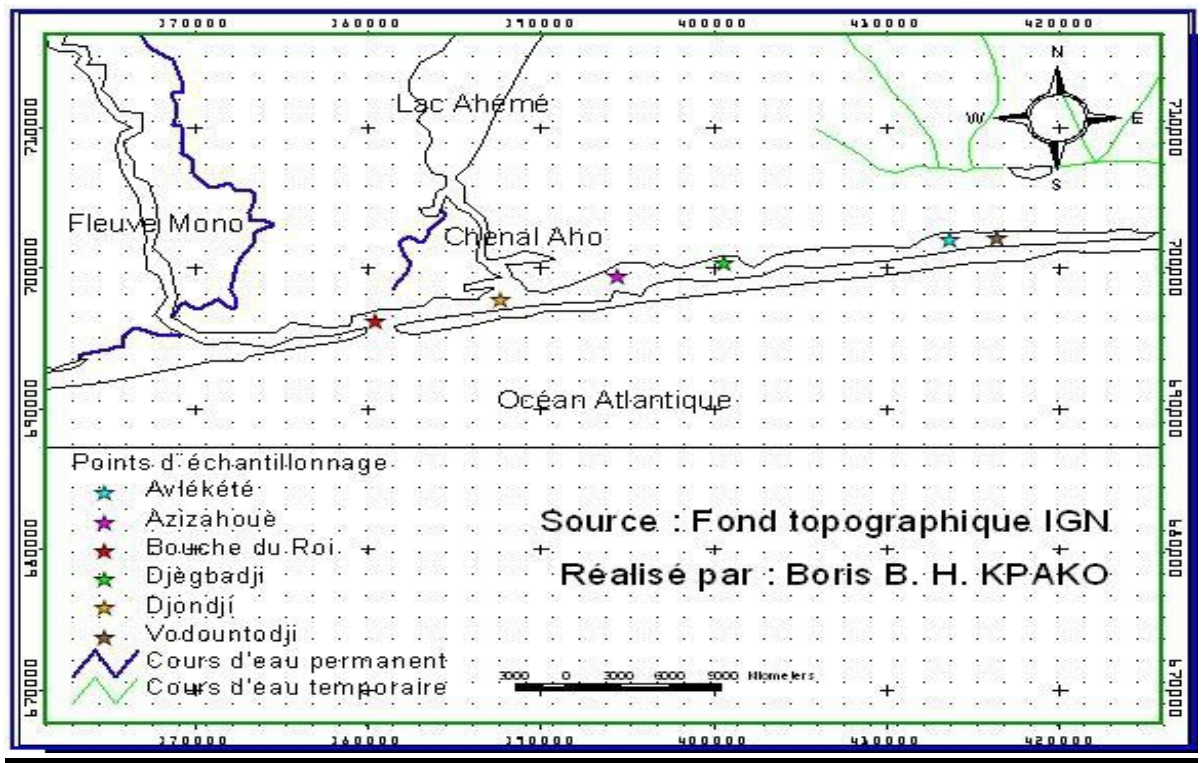

Figure 1. Localisation de la zone d'étude et des points d'échantillonnage

\section{Procédure du dosage des métaux}

Le dosage des métaux a été effectué avec un spectrophotomètre HACH LANGE DR2800. Le cuivre est déterminé par la méthode de Bicinchroninate et le zinc par la méthode de Zincover.

\section{Procédure du test de cytotoxicité sur l'Allium cepa}

La méthode utilisée pour l'étude de toxicité est naturelle et se base sur l'ensemencement d'oignons dans les eaux échantillonnées à diverses proportions données. La réalisation d'un test de toxicité nécessite une certaine quantité d'eau distillée et d'eau échantillonnée. Le test de cytotoxicité a été effectué au Laboratoire de Chimie de la Faculté des Sciences et Techniques à l'Université d'Abomey-Calavi et se repose sur l'inhibition de la croissance des longueurs racinaires des oignons exposés pendant 96 heures dans différentes dilutions des eaux prélevées sur les 06 sites. On dispose donc de 25 verres jetables suivant les six (06) stations de prélèvement ; donc au total cent-cinquante (150) verres jetables. Les 25 verres de chaque site sont répartis suivant 5 gammes de concentration contenant respectivement : $100 \%$ d'eau distillée servant de témoin; $25 \%$ d'eau de la lagune et $75 \%$ d'eau distillée; $50 \%$ d'eau de la lagune et $50 \%$ d'eau distillée; $75 \%$ d'eau de la lagune et $25 \%$ d'eau distillée; $100 \%$ d'eau de la lagune à analyser. Chaque concentration comporte cinq (5) essais. Les verres sont numérotés comme suit : "Concentration d'échantillon, Site, Essai » (exemple : 0\%, S1, E1). On cultive l'oignon dans chacun des verres jetables après avoir enlevé les racines et la peau sèche. Les différentes 
dilutions utilisées pour le test de cytotoxicité sont remplacées toutes les 24 heures avec de nouvelles solutions diluées pendant $72 \mathrm{~h}$. A la fin des 4 jours d'exposition, les longueurs des racines des oignons ont été mesurées par concentration et leurs moyennes ont été calculées. Les sites qui présentent des oignons à racines très atrophiées à $100 \%$ d'échantillon indiquent des eaux à toxicité aigüe.

\section{Analyse des données}

Les données statistiques recueillies sur le terrain et celles issues du dosage des éléments traces métalliques ont été traitées à l'aide du logiciel Microsoft Excel 2016. L'inhibition de la croissance des racines a été estimée par $\mathrm{CE}_{50}$ (Concentration Efficace pour laquelle les effets chimiques sont observés pour $50 \%$ des individus testés). Les concentrations efficaces $\mathrm{CE}_{50}$ et les équations de régression sont déterminées à partir du tracé de la courbe de la longueur des racines en fonction des pourcentages des concentrations des échantillons d'eau en utilisant le programme Microsoft Excel 2016. L'Analyse en Composante Principale (ACP) ainsi que la matrice de corrélation ont été effectuées par le logiciel XLSTAT version 2013.3.02.

\section{Resultats}

\section{Paramètres physico-chimiques}

- Température: Dans notre zone d'étude, la température ne présente pas de grandes variations allant d'une source à une autre, mais néanmoins, elle présente des valeurs plus ou moins élevées avec un minimum de $29.8^{\circ} \mathrm{C}$ obtenu à Avlékété et un maximum de $31,7^{\circ} \mathrm{C}$ enregistré à Djègbadji (Figure 2).

- $\quad$ Potentiel d'hydrogène: Le $\mathrm{pH}$ des eaux analysées varie de 7,2 à 7,53 et donc est presque uniforme sur la lagune (Figure 2). Il appartient à l'intervalle $[6,5 ; 9,5]$ correspondant à la directive fixée par l'Organisation Mondiale de la Santé (OMS) pour les eaux de surface. Ce qui permet de conclure que les valeurs obtenues sont dans la limite du tolérable.

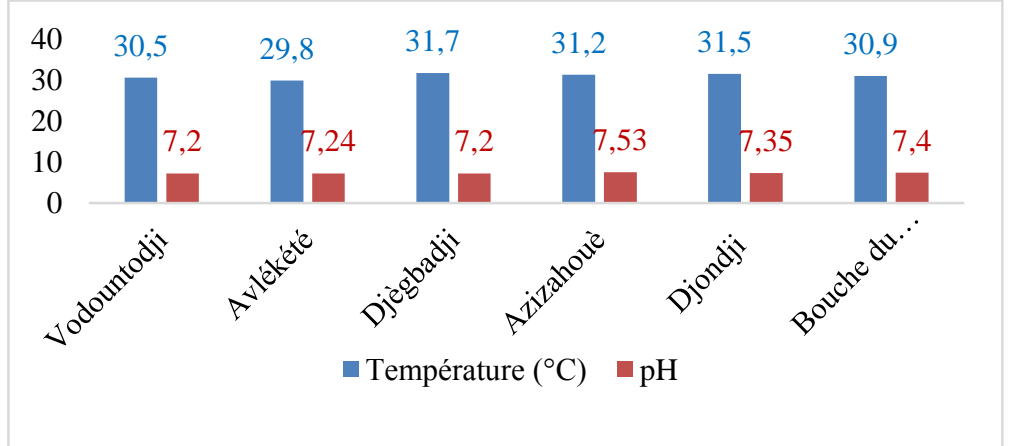

Figure 2. Variation des valeurs de Température et de Potentiel d'Hydrogène 
- Conductivité: Les eaux de la lagune côtière sont fortement minéralisées, avec des valeurs comprises entre 7,83 et $15,49 \mathrm{mS} / \mathrm{cm}$ largement supérieures à l'intervalle admise pour les eaux naturelles soit $[0,05$ $; 1,5] \mathrm{mS} / \mathrm{cm}$. Le pic a été enregistré à Djègbadji suivi d'une baisse progressive de ces valeurs jusqu'à l'embouchure (Figure 3).

- TDS: La variation spatiale des valeurs de TDS présente la même allure que celui de la conductivité avec un minimum de $7,04 \mathrm{~g} / \mathrm{L}$ obtenu à l'embouchure et un maximum de 15,63g/l obtenu à Djègbadji (Figure 3).

- $\quad$ Salinité: Tout comme les graphes de conductivité et de TDS, le graphe de la salinité présente aussi la même allure avec un minimum de 3,9 $\mathrm{mg} / \mathrm{L}$ obtenu à l'embouchure et un maximum de $9,2 \mathrm{mg} / \mathrm{L}$ obtenu à Djègbadji (Figure 3).

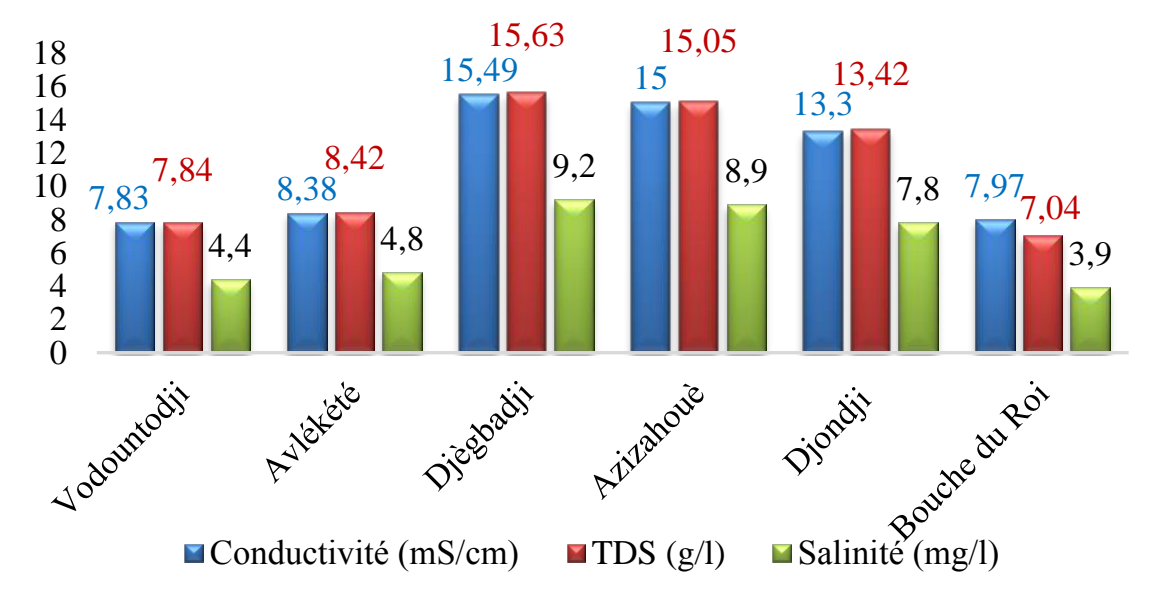

Figure 3. Variation des valeurs de Conductivité, de TDS et de Salinité

- Potentiel redox : Les valeurs du potentiel redox sont inférieures à $25 \mathrm{mV}$ et varient très peu avec un minimum de $-32,5 \mathrm{mV}$ obtenu à l'embouchure et un maximum de $-26 \mathrm{mV}$ obtenu à Djondji (Figure 4). On pourra souligner que la lagune présente globalement les caractères d'un milieu réducteur. 


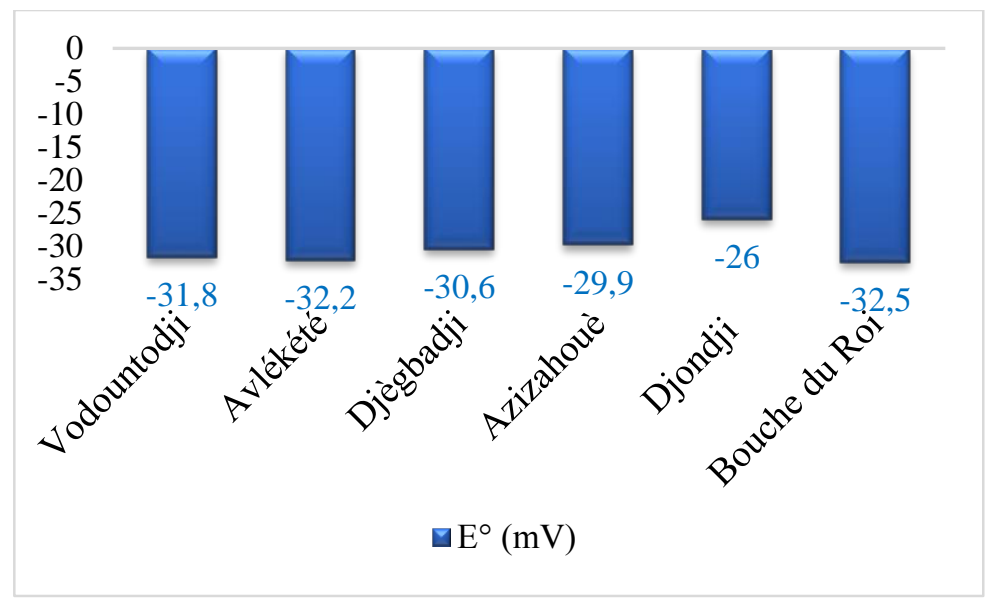

Figure 4. Variation des valeurs de Potentiel redox

\section{Eléments métalliques}

- Cuivre: La Figure 5 nous présente la variation spatiale des concentrations de cuivre dans la lagune côtière. Ces valeurs obtenues varient entre 0,018 et $0,135 \mathrm{mg} / \mathrm{L}$ et sont tous au-dessous de la limite admise par la norme canadienne pour les Critères de Prévention de la Contamination selon les Organismes aquatiques uniquement (CPC_O) « soit $38 \mathrm{mg} / \mathrm{L}$ » ainsi que pour les Critères de Prévention de la Contamination selon la qualité de l'Eau et les Organismes aquatiques (CPC_EO) « soit $1 \mathrm{mg} / \mathrm{L}$ ». Mais, elles sont aux dessus de la limite admise par la norme canadienne pour les Critères de protection de la Vie Aquatique selon des effets Chroniques (CVAC) « soit $0,0085 \mathrm{mg} / \mathrm{L}$ » ainsi que pour les Critères de protection de la Vie Aquatique selon des effets Aigus (CVAA) «soit $0,0127 \mathrm{mg} / \mathrm{L}$ ». Nous pouvons déjà souligner que les concentrations de cuivre dans la lagune côtière du Bénin ne sont pas dans la limite tolérable pour la survie des organismes aquatiques que ce soit selon les effets aigus ou chroniques mais elles sont acceptables pour la consommation de ces organismes aquatiques par l'Homme et même comme eau de boisson après avoir contrôlé les autres paramètres. $\mathrm{Ne}$ constituant pas un risque pour l'Homme, ces valeurs obtenues peuvent néanmoins empiéter sur la disponibilité des organismes vivants dans la lagune. Les valeurs élevées de cuivre ont été enregistrées à Djondji et dans une moindre mesure à Azizahouè et à la Bouche du Roi. 


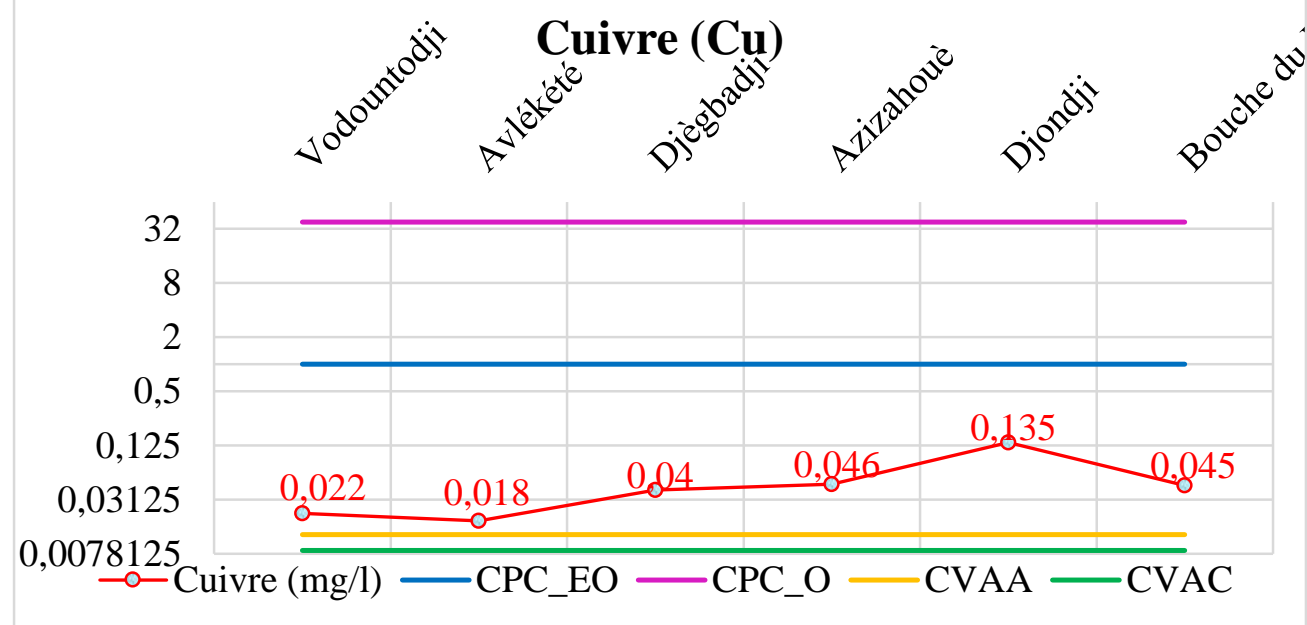

Figure 5. Variation des valeurs de Cuivre

- $\quad$ Zinc : Les concentrations de zinc obtenues sur la lagune côtière varient de 0,12 à $0,2 \mathrm{mg} / \mathrm{L}$ (Figure 6). Tout comme le cuivre, ces valeurs sont en dessous du CPC_O « soit $26 \mathrm{mg} / \mathrm{L}$ » et du CPC_EO « soit $5 \mathrm{mg} / \mathrm{L}$ » et audessus du CVAC « soit $0,11 \mathrm{mg} / \mathrm{L}$ » et du CVAA « soit $0,11 \mathrm{mg} / \mathrm{L}$ ». Les concentrations de zinc ne constituent donc par un risque pour l'Homme ; mais elles représentent un risque pour les organismes présents dans la lagune. Le pic a été enregistré à l'embouchure.

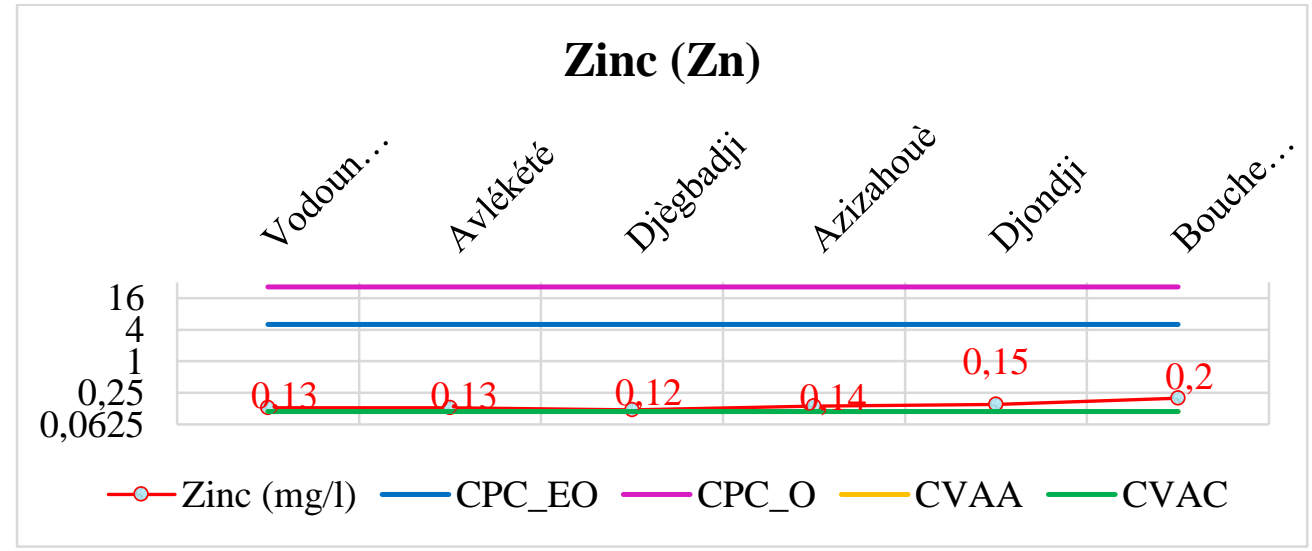

Figure 6. Variation des valeurs de Zinc

\section{Test de cytotoxicité sur l'oignon} 1.

Les résultats issus du test de toxicité sont consignés dans le Tableau 
Tableau 1. Moyenne ( \pm DS*) des longueurs des racines exposées dans les différentes concentrations de chaque site d'échantillonnage en centimètre.

\begin{tabular}{ccccccc}
\hline & Vodountodji & Avlékété & Djègbadji & Azizahouè & Djondji & Bouche du Roi \\
\hline $\mathbf{0 \%}$ & $2,09 \pm 1,31$ & $2,78 \pm 1,80$ & $3,88 \pm 1,55$ & $1,09 \pm 0,89$ & $3,07 \pm 1,14$ & $2,09 \pm 1,17$ \\
\hline $\mathbf{2 5 \%}$ & $2,75 \pm 1,75$ & $2,97 \pm 1,70$ & $2.03 \pm 1,62$ & $0,70 \pm 0,64$ & $0,62 \pm 0,50$ & $2,02 \pm 0,81$ \\
\hline $\mathbf{5 0 \%}$ & $1,71 \pm 1,11$ & $1,14 \pm 0,81$ & $0,31 \pm 0,49$ & $0,53 \pm 0,64$ & $1,49 \pm 1,19$ & $2,96 \pm 1,49$ \\
\hline $\mathbf{7 5 \%}$ & $2,34 \pm 2,65$ & $0,71 \pm 0,69$ & $1,69 \pm 1,63$ & $0,55 \pm 0,44$ & 0 & $1,79 \pm 1,87$ \\
\hline $\mathbf{1 0 0 \%}$ & $1,28 \pm 1,64$ & $1 \pm 0,60$ & $0,14 \pm 0,22$ & $0,32 \pm 0,26$ & $0,11 \pm 0,18$ & $0,7 \pm 0,84$ \\
\hline
\end{tabular}

*DS : Déviation Standard (Écart- type)

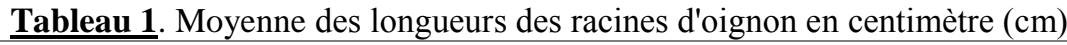

\begin{tabular}{cccccc}
\hline Sites & $0 \%$ & $25 \%$ & $50 \%$ & $75 \%$ & $100 \%$ \\
\hline S1 & 2,09 & 2,75 & 1,71 & 2,34 & 1,28 \\
\hline S2 & 2,78 & 2,97 & 1,14 & 0,71 & 1 \\
\hline S3 & 3,88 & 2,03 & 0,31 & 1,69 & 0,14 \\
\hline S4 & 1,09 & 0,70 & 0,53 & 0,55 & 0,32 \\
\hline S5 & 3,07 & 0,62 & 1,49 & 0 & 0,11 \\
\hline S6 & 2,09 & 2,02 & 2,96 & 1,79 & 0,7
\end{tabular}

Les courbes Dose-Réponses obtenus sur les différentes stations de prélèvement pour la détermination des concentrations efficaces $\left(\mathrm{CE}_{50}\right.$ : concentration qui induit cinquante pourcents d'effet) sont présentées sur la Figure 7.
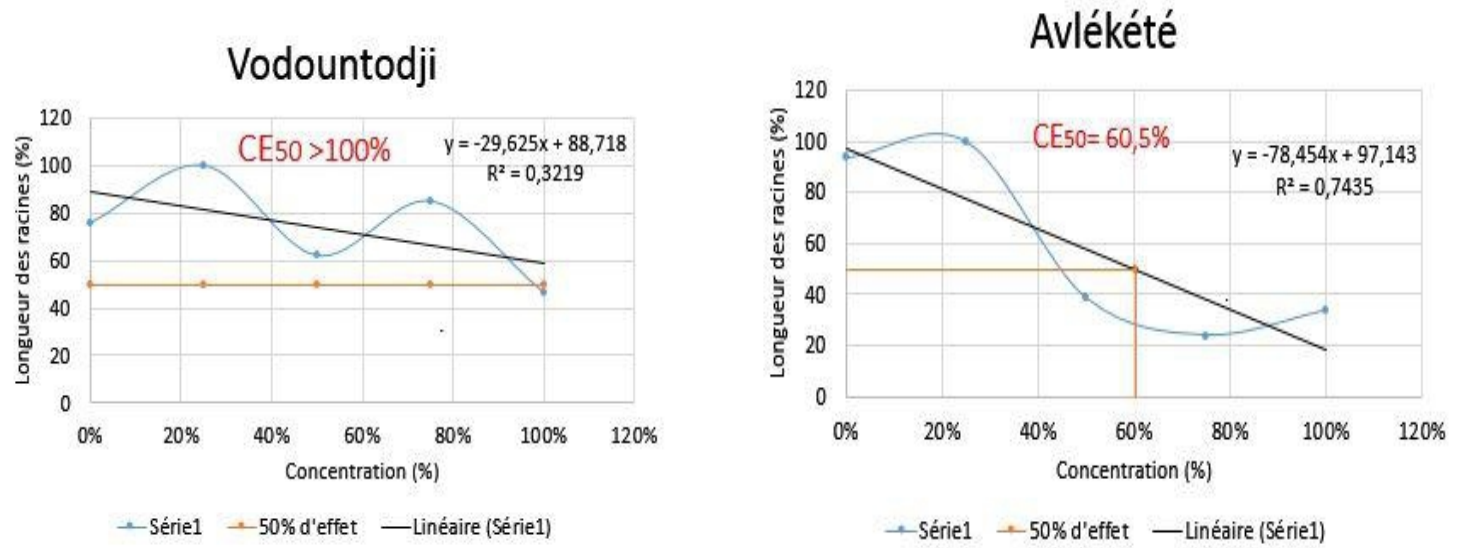
Djègbadji

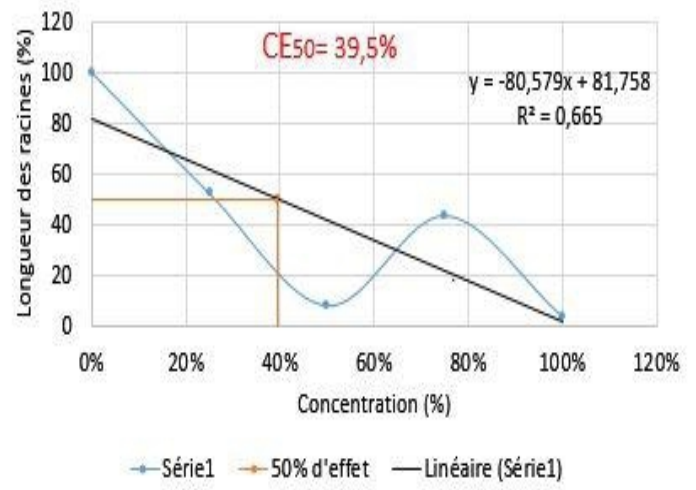

Djondji

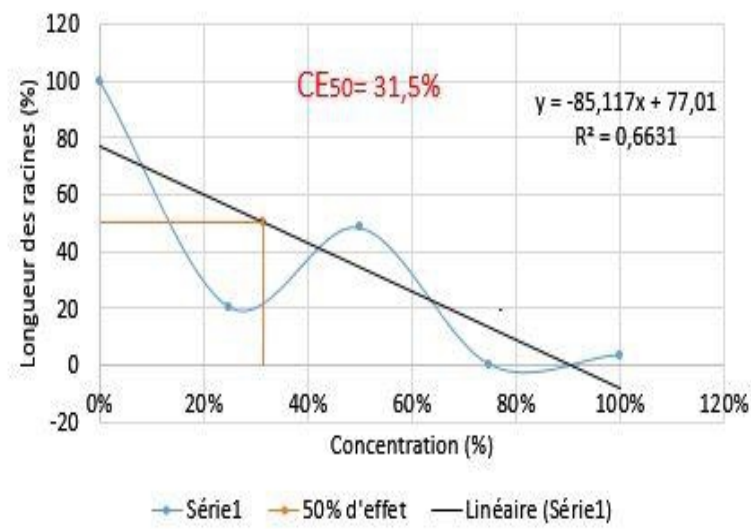

Azizahouè

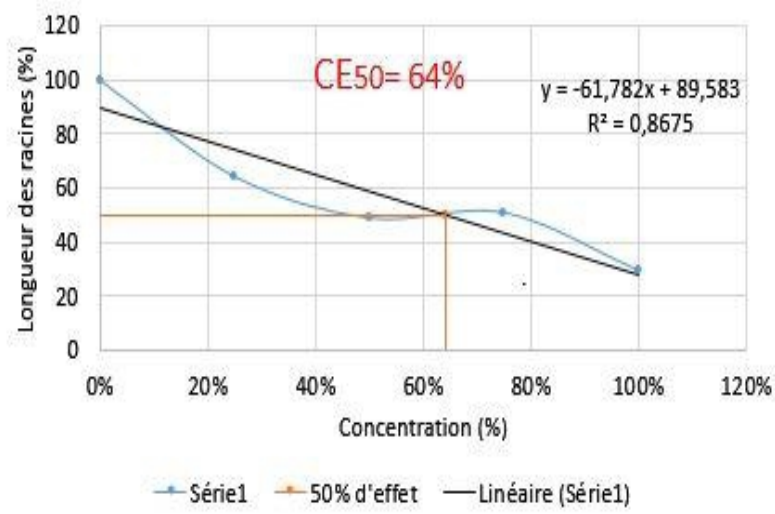

Bouche du Roi

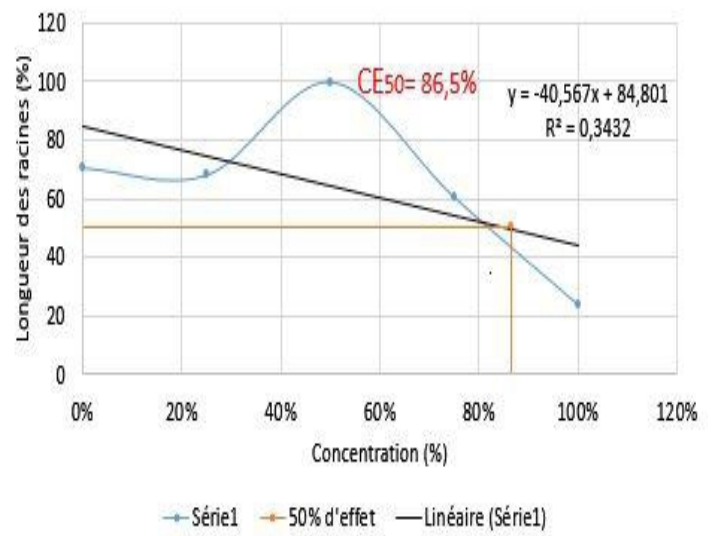

Figure 7. Courbes Dose-Réponse pour l'évaluation de la cytotoxicité

A partir des données de la figure 7 , on remarque que les racines d'oignon exposées à $100 \%$ d'échantillon sont atrophiées par rapport aux racines témoins à $0 \%$ d'échantillon, ce qui traduit que les sites sont pollués. Ceci se confirme également par la décroissance des différentes droites de régression linéaire qui, quant à elles, représente bien la distribution des points d'échantillonnage avec un coefficient de détermination $\mathrm{R}^{2}>60 \%$ dans certains cas (Avlékété, Djègbadji, Azizahouè et Djondji) et dans d'autre cas, n'explique pas la distribution de ces points (Vodountodji et Bouche du Roi) avec un $\mathrm{R}^{2}<50 \%$.

Ensuite, les concentrations efficaces qui induisent cinquante pourcent d'effet $\left(\mathrm{CE}_{50}\right)$ obtenues à partir des graphes ont permis de classer le degré de toxicité des sites d'échantillonnage. Plus cette concentration petite et plus le 
site est plus pollution. Ceci nous à classer l'échelle de toxicité des différents sites de prélèvement par ordre décroissant : Djondji - Djègbadji - Avlékété Azizahouè - Bouche du Roi - Vodountodji.

Le Tableau 2 nous présente 1'échelle de toxicité des différents sites de prélèvement par ordre décroissant (du plus toxique au moins toxique) en se basant sur les concentrations efficaces qui induisent cinquante pourcents d'effet (CE50).

Tableau 2. Gradient de toxicité des stations de prélèvement

\begin{tabular}{ccc}
\hline \multirow{2}{*}{ STATIONS } & $\begin{array}{c}\text { Concentration Efficace } \mathrm{CE}_{50} \\
(\%)\end{array}$ & $\begin{array}{c}\text { Coefficients de détermination } \mathrm{R}^{2} \\
(\%)\end{array}$ \\
\hline Djondji & 31,5 & 66 \\
\hline Djègbadji & 39,5 & 67 \\
\hline Avlékété & 60,5 & 74 \\
\hline Azizahouè & 64 & 87 \\
\hline Bouche du Roi & 86,5 & 34 \\
\hline Vodountodji & $>100$ & 32 \\
\hline
\end{tabular}

D'après le Tableau 2, on remarque que la Bouche du Roi et Vodountodji présentent de faible valeur de coefficient de détermination (respectivement $34 \%$ et $32 \%$ ) accordant donc un minimum de doute aux concentrations efficaces obtenues. L'Analyse en Composante Principale (ACP) nous permettra par la suite de confirmer la position de ces deux sites dans ledit Tableau.

\section{Analyse en Composante Principale (ACP)}

- Matrice de corrélation: Le Tableau 3 présente une matrice de corrélation de Pearson montrant les valeurs des coefficients de détermination $\mathrm{R}^{2}$ pour un seuil de rejet de $5 \%$. L'analyse dudit tableau montre une forte corrélation positive entre la salinité, la conductivité et le TDS. Ceci implique que ces trois paramètres évoluent de façon proportionnelle. Il en est de même pour le cuivre et le potentiel redox qui présente aussi une corrélation positive. P100 est fortement et négativement corrélé avec la température, la conductivité, les Solides Totaux Dissous (TDS) et la salinité. Ce qui stipule que P100 est inversement proportionnel aux paramètres de minéralisation. C'est-à-dire que quand l'eau est riche en minéraux dont les sels (eau salée), les racines sont plus atrophiées à $\mathrm{P} 100$ et vice-versa.

Tableau 3. Matrice de corrélation

\begin{tabular}{cccccccccccccc}
\hline Var & $\Theta$ & Cond & TDS & Salinité & $\mathrm{pH}$ & $\mathrm{E}^{\circ}$ & Cuivre & Zinc & P0 & P25 & P50 & P75 & P100 \\
\hline$\theta$ & $\mathbf{1}$ & 0.80 & 0.76 & 0.76 & 0.26 & 0.61 & 0.59 & 0.04 & 0.26 & -0.73 & -0.27 & -0.14 & $\mathbf{- 0 . 8 6}$ \\
Cond & & $\mathbf{1 . 0 0}$ & $\mathbf{1 . 0 0}$ & $\mathbf{1 . 0 0}$ & 0.30 & 0.61 & 0.41 & -0.38 & 0.21 & -0.71 & -0.75 & -0.44 & $\mathbf{- 0 . 8 9}$ \\
TDS & & & $\mathbf{1}$ & $\mathbf{1 . 0 0}$ & 0.25 & 0.62 & 0.40 & -0.46 & 0.23 & -0.68 & -0.80 & -0.45 & $\mathbf{- 0 . 8 5}$ \\
Sal & & & & $\mathbf{1}$ & 0.25 & 0.61 & 0.39 & -0.46 & 0.22 & -0.68 & -0.80 & -0.46 & $\mathbf{- 0 . 8 5}$ \\
pH & & & & & $\mathbf{1}$ & 0.25 & 0.30 & 0.50 & -0.71 & -0.74 & 0.09 & -0.48 & -0.39 \\
$\mathrm{E}^{\circ}$ & & & & & & $\mathbf{1}$ & $\mathbf{0 . 9 3 1}$ & -0.12 & 0.21 & -0.80 & -0.27 & -0.71 & -0.70
\end{tabular}




\begin{tabular}{|c|c|c|c|c|c|c|c|}
\hline $\mathrm{Cu}$ & $\mathbf{1}$ & 0.21 & 0.22 & -0.77 & 0.07 & -0.65 & -0.66 \\
\hline $\mathrm{Zn}$ & & 1 & -0.33 & -0.18 & 0.85 & 0.06 & -0.00 \\
\hline P0 & & & 1 & 0.22 & -0.23 & 0.01 & -0.31 \\
\hline P25 & & & & 1 & 0.20 & 0.65 & 0.80 \\
\hline P50 & & & & & 1 & 0.31 & 0.42 \\
\hline P75 & & & & & & 1 & 0.55 \\
\hline P100 & & & & & & & 1 \\
\hline
\end{tabular}

Les valeurs en gras sont différentes de 0 à un niveau de signification alpha $=0,05$

Cercle de corrélation: La Figure 8 nous présente le cercle de corrélation entre tous les variables explicatives.

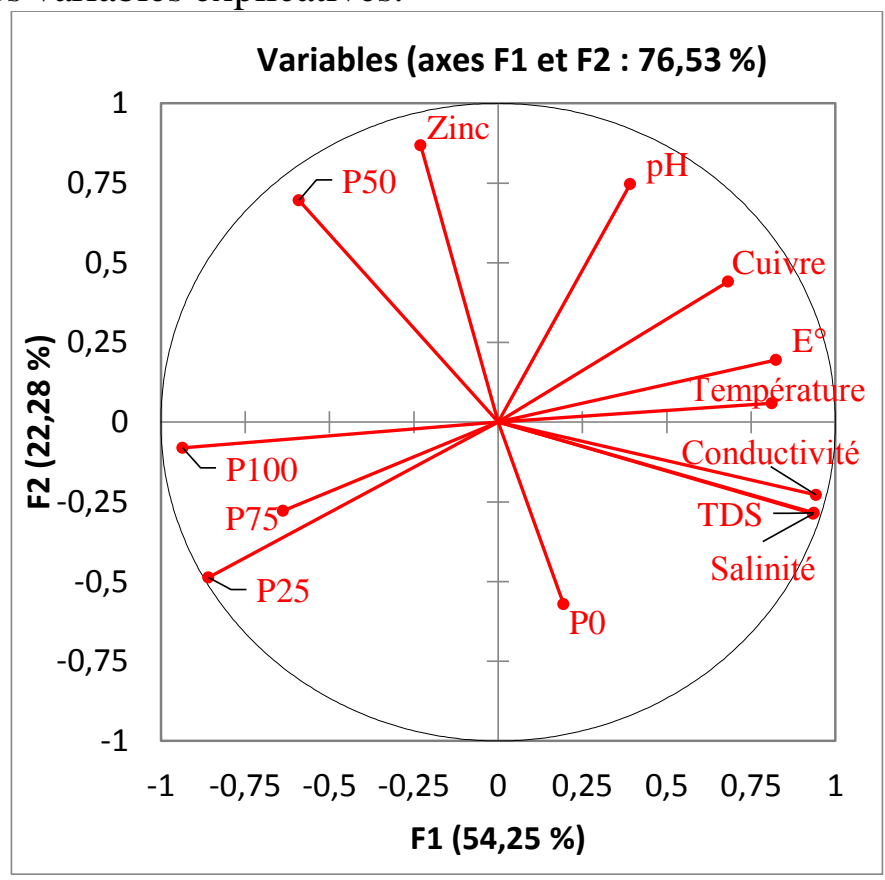

Figure 8. Cercle de corrélation des variables explicatives

Toutes les variables explicatives sont généralement proches du cercle de corrélation sauf $\mathrm{P} 0$ et $\mathrm{P} 75$. Ces variables sont donc bien représentées. Mais P0 et P75 le sont dans une moindre mesure. La conductivité, le TDS et la salinité présentent non seulement une très forte corrélation positive entre eux mais aussi avec l'axe F1 (encore appelé facteur F1 ou composante principale F1). Ceci nous permet de conclure que l'axe F1 oppose les sites très minéralisés aux sites peu minéralisés. Ce même axe oppose les variables P100, P75, P50, P25 à la variable P0. Ce qui signifie que l'axe F1 différencie aussi les oignons à racines moins développées en présence d'échantillon (P100, P75, P50 et P25) à celles développées uniquement en présence de l'eau distillée ( $\mathrm{P} 0)$, ce qui, dans notre cas, équivaut à l'opposition des eaux toxiques aux eaux toxiques. Quant à l'axe F2, il oppose les eaux riches en 
métaux (zinc et cuivre) avec une corrélation positive aux eaux qui en sont pauvres.

Répartition des sites combinée aux variables : La Figure 9 nous présente la répartition des sites d'échantillonnage combinée à celle des variables explicatives.

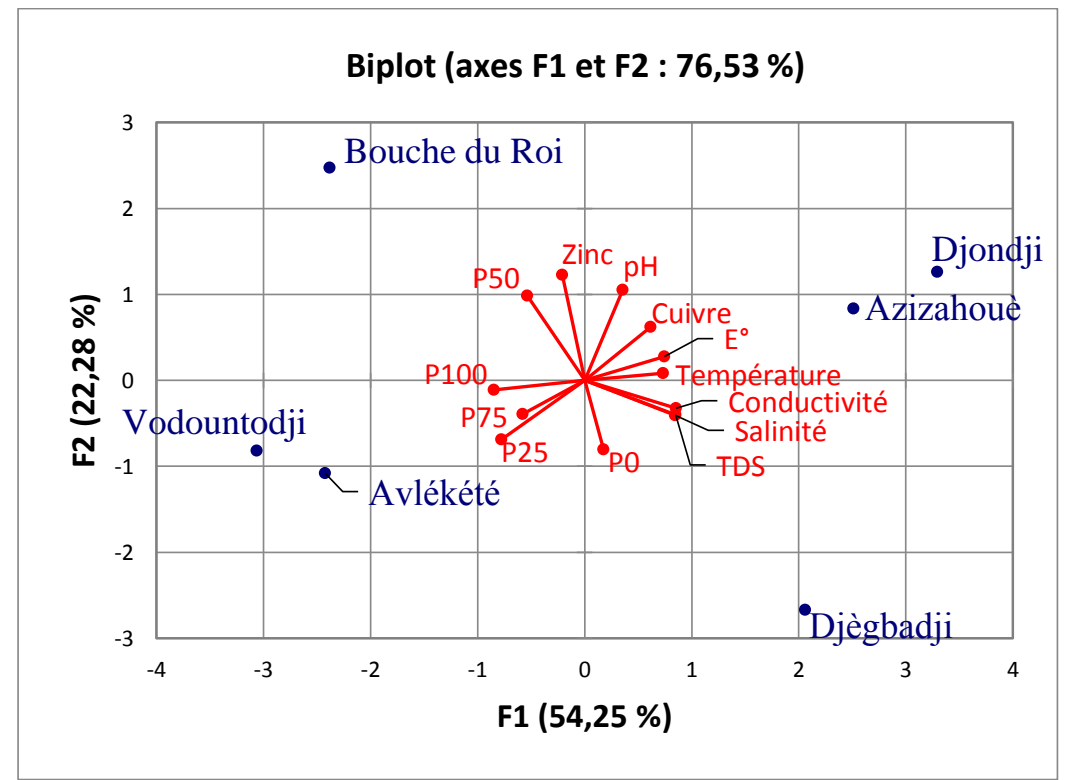

Figure 9. Répartition des sites d'échantillonnage sur le graphe factoriel

De l'analyse de la Figures 9, nous pouvons conclure que:

- L'embouchure de la lagune côtière est riche en zinc et en cuivre et est constituée d'eau très pauvre en minéraux. Ces eaux sont très peu toxiques avec le test sur l'Allium cepa.

- L'ouverture du lac Ahémé sur la lagune côtière par le chenal Aho à Djondji présente des eaux riches en cuivre, en zinc et en minéraux. Ces eaux présentent une toxicité aiguë avec la méthode sur l'Allium cepa.

- Tout comme Djondji, Azizahouè est constitué d'eau riches en cuivre, en zinc et en minéraux et présente aussi une toxicité aiguë avec le test sur l'oignon dans une moindre mesure car elle est mal représentée (proche du centre) comparativement à Djondji.

- A Djègbadji, les eaux sont pauvres en cuivre et en zinc mais très riches en minéraux. Le test sur l'Allium cepa montre que ces eaux sont très toxiques.

- $\quad$ Tout comme Vodountodji, Avlékété présente des eaux pauvres en zinc, en cuivre et en minéraux et aussi très peu toxiques. Vodountodji est moins toxique que Avlékété parce qu'il est très bien représenté sur le graphe que celui-ci. 
- Vodountodji présente des eaux pauvres en zinc, en cuivre et en minéraux. Avec le test sur l'Allium cepa, ces eaux se sont avérées très peu toxiques. Il faut remarquer que le site Vodountodji est un site très protégé suivant les croyances locales. Vodountodji est une zone protégée où personne ne pratique la pêche à cause des divinités de la localité d'où une faible anthropisation.

\section{Discussion}

Les valeurs élevées observées pour la mesure de la température peuvent être expliquées par l'effet du réchauffement climatique sur la lagune côtière puisse qu'aucune source de rejet d'eau résiduelle chaude n'a été enregistrée. Cette explication fait référence à l'étude de Boko et al. (2012) sur l'enjeu du changement climatique au Bénin soulignant que la température s'est élevée de $0,9^{\circ} \mathrm{C}$ après 2010 .

Le graphe de la conductivité, du TDS et de la salinité présente les mêmes allures, ce qui signifie une forte corrélation entre ces paramètres. Cette corrélation se confirme avec la matrice de corrélation. La forte corrélation entre TDS, salinité et conductivité s'explique par la dissolution des substances chimiques dans l'eau, enrichissant ainsi le milieu en ion (exemple : $\mathrm{Na}+, \mathrm{Cl}-\ldots)$. Ces ions participent ensuite à la formation de sel et augmente aussi la capacité de l'eau à conduire le courant électrique. Le pic obtenu à Djègbadji suivi d'une décroissance sur le graphe de la salinité est dû à l'accumulation du sel causé par l'intrusion saline autre fois dans la période de haute marée. En période de basse marrée (Novembre 2016), le phénomène tend à s'inverser et le lac Ahémé apporte une quantité importante d'eau vers la lagune à Djondji par l'intermédiaire du chenal Aho qui coule vers l'embouchure ainsi que l'apport du fleuve Mono à l'embouchure. La très forte valeur obtenue à Djègbadji est aussi due à la production abondante de sel. Il faut également noter que la minéralisation des eaux de la lagune côtière est principalement due aux sels. Les valeurs élevées de salinité obtenues par la présente étude sont en accord avec celles de Adandedjan D., (2012) stipulant que la salinité de l'eau sur la lagune côtière est élevée avec une moyenne de $5,28 \mathrm{mg} / \mathrm{L}$.

Les fortes valeurs d'éléments traces métalliques (cuivre et de zinc) obtenues à Djondji et à la Bouche du Roi sont respectivement dues à l'apport de lac Ahémé et du fleuve Mono. Les résultats obtenus indiquent donc que les eaux de la lagune côtière sont polluées par le cuivre et le zinc. Il en est de même pour les eaux du lac Nokoué car Lawani en 2013 a trouvé des valeurs allant jusqu'à $0,51 \mathrm{mg} / \mathrm{l}$ pour le cuivre et $0,239 \mathrm{mg} / \mathrm{l}$ pour le zinc. Le calcul des indices de contamination par le cuivre et le zinc fait ressortir un indice supérieur aux normes admises et classe la lagune côtière comme une zone de classe 2 (polluée) pour le cuivre et de classe 1 pour le zinc (non pollué). Ce 
qui conduit à la conclusion suivante : le sédiment superficiel de ce plan d'eau est pollué par le cuivre avec un risque moyen principalement au niveau du site de Hio. Cette concentration élevée du cuivre dans les eaux résulte de la mauvaise gestion des déchets (liquides et solides) par les populations et des eaux usées provenant des toilettes installées sur la bordure de la lagune. La corrélation positive entre le cuivre et le potentiel redox relate une augmentation proportionnelle de ces deux paramètres. C'est-à-dire que, plus le milieu est oxygéné, plus la quantité de cuivre dissoute dans l'eau est importante. Ceci nous amène à affirmer que l'ion cuivrique $\left(\mathrm{Cu}^{2+}\right)$ domine ces eaux car il est plus abondant dans les eaux oxygénées et moins abondant quand le milieu devient réducteur laissant place à l'ion cuivreux $\left(\mathrm{Cu}^{+}\right)$qui s'avère abondant dans les eaux anoxiques.

Les coefficients de détermination $\left(\mathrm{R}^{2}\right)$ faible obtenus à Vodountodji et à la Bouche du Roi sont dus aux erreurs dans le protocole utilisé pour l'évaluation de la cytotoxicité des eaux. Il s'agit principalement de la non homogénéité des oignons. Les concentrations efficaces CE50 obtenus par les courbes Dose-Réponse permettent d'affirmer que Djondji est le site le plus pollué $\left(\mathrm{CE}_{50}=31,5 \%\right)$, ensuite viennent Djègbadji $\left(\mathrm{CE}_{50}=39,5 \%\right)$, Avlékété $\left(\mathrm{CE}_{50}=60,5 \%\right)$, Azizahouè $\left(\mathrm{CE}_{50}=64 \%\right)$, Bouche du Roi $\left(\mathrm{CE}_{50}=86,5 \%\right)$ et enfin Vodountodji $\left(\mathrm{CE}_{50}>100 \%\right)$. L'ACP nous a permis de confirmer la position des deux derniers sites (avec un $\mathrm{R}^{2}$ faible) dans la classification du niveau de toxicité. D'après la Figure 9, les deux sites restants (Bouche du Roi et Vodountodji) sont très peu toxiques et pauvres en minéraux ; néanmoins l'embouchure est riche en zinc et en cuivre contrairement à Vodountodji. On peut donc d'abord déduire que les eaux de l'embouchure sont plus toxiques que celles de Vodountodji. Cela se confirme, du fait que Vodountodji est une zone protégée où personne ne pratique la pêche à cause des divinités de la localité et donc une faible anthropisation. Les résultats nous montrent que le test de toxicité sur l'oignon est non seulement influencé par les métaux (cuivres et zinc) mais aussi par la salinité de l'eau. Les eaux très salées se sont avérées toxiques même avec une faible concentration en métaux (exemple : Djègbadji). Cela se confirme par le fait que la matrice de corrélation stipule que : si la salinité, la conductivité et le TDS augmentent, alors P100 diminue. Or une faible valeur de P100 signifie que les racines d'oignon sont atrophiées à $100 \%$ d'échantillon contrairement au témoin (P0) ce qui amène à juger le site concerné de pollué. Donc si le site est jugé toxique quand P100 diminue alors, au moment où ce site sera aussi très salé, il sera jugé toxique. On pourra donc classer l'embouchure au-dessous des sites à la fois minéralisés et riches en métaux. La toxicité remarquable enregistrée à Avlékété malgré la faible concentration en sel, en cuivre et en zinc nous amène à soupçonner la présence d'un autre type de micropolluant non prise en compte dans cette étude. La classification par ordre décroissant 
de toxicité des sites se confirme donc et on a: Djondji, Djègbadji, Avlékété, Azizahouè, Bouche du Roi et Vodountodji. L'uniformité décroissante des droites de régression obtenus sur les différents sites spécifiant que les racines d'oignon sont atrophiées à $100 \%$ par rapport au témoin $(0 \%)$ est en accord avec la conclusion de Cakpo, A. et al. (2015) à partir des résultats des longueurs racinaires d'oignon obtenus grâce au test de cytotoxicité sur les sites d'Accron et du Pont de la lagune de Porto-Novo. Lesdits résultats montrent que ces deux sites sont tous toxiques mais celui du Pont en est plus que celui d'Accron.

Il sera aussi recommandé d'utiliser le test de cytotoxicité avec l'oignon sur des eaux dont la salinité est faible pour remédier à l'interférence de la salinité et des éléments toxiques (les métaux) et aussi de cultiver soimême les oignons sur un terrain homogène en termes de richesse en nutriment afin d'obtenir un résultat uniforme. Il sera aussi préférable d'utiliser de l'eau synthétique dont la composition est : $60 \mathrm{mg} / \mathrm{L}$ de $\mathrm{CaSO}_{4}$, 60mg/L de $\mathrm{MgSO}_{4}, 96 \mathrm{mg} / \mathrm{L}$ de $\mathrm{NaHCO}_{4}$ et $4 \mathrm{mg} / \mathrm{L} \mathrm{KCl}$ (Rank \& Nielsen, 1997) à la place de l'eau distillée pour pourvoir augmenter la poussée des racines du témoin, qui, est sensée se rivaliser avec un échantillon prélevé contenant naturellement certains sels minéraux bénéfiques à l'Allium cepa.

\section{Conclusion}

Les eaux de la lagune côtière ne constituent pas un biotope adéquat pour la protection des organismes aquatiques qui y sont présents. La rareté des poissons soulignée par les pêcheurs sur la lagune serait en partie due à la concentration élevée des polluants tels que le cuivre et le zinc étudiés dans ce travail et confirme également l'appréciation des résultats de cytotoxicité sur l'Allium cepa stipulant que les organismes aquatiques sont exposés aux effets toxiques des micropolluants. L'ouverture du fleuve Mono sur la lagune côtière ainsi que celle du lac Ahémé par le chenal Aho constituent les principales sources d'apport en éléments traces métalliques dans la lagune. Ces résultats montrent l'urgence d'une intervention rapide en amont de la lagune côtière en adoptant une politique de contrôle du rejet des eaux usées dans le fleuve Mono et le lac Ahémé.

\section{References:}

1. Adandedjan, D., Laleye, P. \& Gourene, G. (2012). Macroinvertebrates communities of a coastal lagoon in southern Benin, West Africa. International Journal of Biological and Chemical Sciences, 6(3) :1233-1252.

2. Adrian, S., Delaume, I., Loblein, M. \& Rumelhard, A. (2006). Les produits toxiques : les peintures toxiques, les produits sanitaires et 
pesticides. Projet, Bassez-Muguet M-P. IUT Robert Schuman Département Chimie, 44p.

3. Alassane, A. (2004). Etude hydrogéologique du continental terminal et des formations de la plaine littorale dans la région de Porto Novo (sud du Benin) : identification des aquifères et vulnérabilité de la nappe superficielle. Thèse de doctorat à l'université Cheikh Anta Diop de Dakar, 185p.

4. Ashraf, H. \& Husain, Q. (2010). Studies on bitter gourd peroxidise catalyzed removal of $\mathrm{p}$ bromophenol from wastewater. Desalination, 262 (1-3) : 267-272.

5. Barillet, S. (2007). Toxicocinétique, toxicité chimique et radiologique de l'uranium chez le poisson zèbre. Thèse de doctorat à l'université Paul Verlaine de Metz, 478p.

6. Boko, M., Kosmowski, F. \& Vissin, E. (2012). Les Enjeux du Changement Climatique au Bénin. Konrad-Adenauer-Stiftung, 72p.

7. Cakpo, A., Sagbo, E., Mama, D. \& Soumanou, M. (2015). Évaluation de la cytogénotoxicité des sites de pollution du PONT et d'ACCRON de la lagune de Porto-Novo. Journal of Applied Biosciences 87 : 8076-8084.

8. Cotelle, S. (1999). Etude de la génotoxicité de matrices complexes à l'aide de plantes supérieures. Thèse de doctorat à l'université de Metz, 258p.

9. Fasla, B. (2009). Evaluation du potentiel antimitotique et génotoxique de plantes médécinales et analyse phytochimique. Mémoire de Magistrer à l'Université d'Oran Es-Sénia. 172p.

10. Feretti, D., Zerbini, I., Zani, C., Ceretti, E., Moretti, M. \& Monarca, S. (2007). Allium cepa chromosome aberration and micronucleus test applied to study genotoxicity of extracts from pesticide-treated vegetables and grades. Food Additives and Contaminants, 24 : 561572.

11. Fiogbé, E., Dossou-Yovo, A. \& Ogouwalé, E. (2007). Rapport National sur l'Environnement Marin et Côtier du Bénin. 68p.

12. Foltete, A-S. (2010). Effets génotoxiques et systèmes de détoxication chez Vicia faba (Fabaceae) dans le cadre de l'évaluation des sols pollués. Thèse de doctorat à l'université Paul Verlaine - Metz, 246p.

13. Goujon, E. (2015). Etude de la toxicité de la sulcotrione et de ses produits de photo-dégradation. Thèse de doctorat à l'université Blaise Pascal, 155p.

14. Halmi Sihem, M. (2015). Etude botanique et phytochimique : Approche biologique et pharmacologique d'Opuntia ficus indica. Thèse de doctorat à l'université des frères Mentouri de Constantine, $243 p$. 
15. Lawani, R.A.N. (2013). Evaluation des éléments traces métalliques et spéciation chimique du cuivre et du zinc dans les eaux et sédiments du lac Nokoué. Mémoire de master II en Ecohydrologie. Université d'Abomey-Calavi. 90p.

16. Leme, DM. \& Marin-Morales, MA. (2008). Chromosome aberration and micronucleus frequencies in Allium cepa cells exposed to petroleum polluted water-A case study. Mutation Research, 650 (1) : 80-86.

17. Ma, TH., Xu, ZD., Xu, CG., Mcconnell, H., Rabago, EV., Arreola, GA. \& Zhang, HG. (1995). The improved Allium/Vicia root tip micronucleus assay for clastogenicity of environmental pollutants. Mutation Research, 334 : 185-195.

18. Manar, R. (2008). Effets populationnels du chlordane sur les microcrustacés cladocères daphnia $\mathrm{sp}$. dans une perspective d'évaluation des risques. Thèse de doctorat à l'université Hassan II, Mohammedia - Maroc en cotutelle avec l'université Paul Verlaine, Metz - France, $177 \mathrm{p}$.

19. MDEP (Ministère du Développement Durable, de l'Environnement et des Parcs) (2009). Critères de qualité de l'eau de surface, Direction du suivi de l'état de l'environnement, ministère du Développement durable, de l'Environnement et des Parcs, Québec, ISBN 978-2-55057559-7 (PDF), 506 p. et 16 annexes.

20. Mittergger, H., Da Silva, J., Arenzon, A., Saraiva, C., Fernandes, IC. $\&$ Pegas, JA. (2007). Evaluation of genotoxicity and toxicity of water and sediment samples from a Brazilian stream influenced by tannery industries. Chemosphere 67: 1211-1217.

21. Mustafa, Y. \& Arikan, ES. (2008). Genotoxicity testing of quizalofop-P-ethyl herbicide using the Allium cepa anaphasetelophase chromosome aberration assay. Caryologia, 61 (1) : 45-52.

22. OMD (Objectifs du Millénaire pour le Développement) (2015). Rapport. New York, Nations Unies : Way C., 75p.

23. PGPE (Plan de Gestion de Plan d'Eau) (2008). Complexe fluviolacustre, Lac Ahémé-Chenal Aho-Lagune côtière. Version finale. $38 p$.

24. Rank, J. \& Nielsen, M.H. (1997). Allium cepa anaphase-telophase root tip chromosome aberration assay on $N$-methyl- $N$-nitrosourea, maleic hydrazide, sodium azide, and ethyl methanesulfonate. Mutation Research/Genetic Toxicology and Environmental Mutagenesis. Volume 390, Issues 1-2, 24 April 1997, pp 121-127. 
25. Riffat, A. \& Ahmad, M. (2006). Allium cepa derived EROD as a potential biomarker for the presence of certain pesticides in water. Chemosphere, $62: 527-537$.

26. Villanueva, M. C. S. (2004). Biodiversité et relations trophiques dans quelques milieux estuariens et lagunaires de l'Afrique de l'Ouest : adaptations aux pressions environnementales. Thèse de doctorat à l'Institut National Polytechnique de Toulouse, 272p. 